PROCEEDINGS OF THE

AMERICAN MATHEMATICAL SOCIETY

Volume 137, Number 3, March 2009, Pages 777-785

S 0002-9939(08)09034-5

Article electronically published on October 29, 2008

\title{
ON NOETHERIAN AFFINE PRIME REGULAR HOPF ALGEBRAS OF GELFAND-KIRILLOV DIMENSION 1
}

\author{
GONGXIANG LIU
}

(Communicated by Martin Lorenz)

\begin{abstract}
Let $k$ be an algebraically closed field. In 2007, D.-M. Lu, Q.-S. $\mathrm{Wu}$, and J. J. Zhang asked the following question: Besides the group algebras $k \mathbb{Z}, k \mathbb{D}$ and infinite dimensional prime Taft algebras, are there other noetherian affine prime regular Hopf algebras of GK-dimension 1? In this paper, we give a new one. Another problem posed by $\mathrm{Lu}, \mathrm{Wu}$, and Zhang can also be resolved by this example. Assuming $H$ is a noetherian affine prime regular Hopf algebra of GK-dimension 1 , we show that $\operatorname{gr} H:=\bigoplus_{s>0} J_{i q}^{s} / J_{i q}^{s+1}$, as a Hopf algebra, is isomorphic to an infinite dimensional prime Taft algebra. This gives a characterization of infinite dimensional prime Taft algebras.
\end{abstract}

\section{INTRODUCTION AND PRELIMINARIES}

Although some of the results are valid under weaker hypotheses, we assume for simplicity of the exposition that our ground field $k$ is algebraically closed. All modules are left modules.

A long time ago, we didn't know how to define the left and right integrals of an infinite dimensional Hopf algebra. The pioneering effort in this direction is 6 . In this nice paper, by using homological properties, the authors define the integrals for a large class of infinite dimensional Hopf algebras as follows. We say a Hopf algebra $H$ is $A S$-Gorenstein (where AS stands for Artin and Schelter) if

(AS1) ${ }_{H} H$ has finite injective dimension, say $d$,

(AS2) $\operatorname{dim}_{k} \operatorname{Ext}_{H}^{d}\left({ }_{H} k,_{H} H\right)=1, \operatorname{Ext}_{H}^{i}\left({ }_{H} k,_{H} H\right)=0$ for all $i \neq d$, and

(AS3) the right $H$-module versions of the conditions (AS1, AS2) hold.

Moreover, we say a Hopf algebra $H$ is AS-regular if it is AS-Gorenstein and it has finite global dimension. AS-Gorensteinness seems strange at first glance, but many noetherian Hopf algebras are AS-Gorenstein. For example, every noetherian affine PI Hopf algebra is AS-Gorenstein [12. For an AS-Gorenstein Hopf algebra $H$ of injective dimension $d$, the left homological integral $\int^{l}$ is defined to be the 1-dimensional $H$-bimodule $\operatorname{Ext}_{H}^{d}\left({ }_{H} k, H H\right)$. If $H$ is finite dimensional, the left homological integral agrees with the classical left integral. The right homological integral can be defined similarly. By homological integrals, the authors of [6] generalize the classical result of Larson and Sweedler about finite dimensional

Received by the editors September 25, 2006, and, in revised form, March 25, 2007.

2000 Mathematics Subject Classification. Primary 16W30.

Key words and phrases. Homological integral, Gelfand-Kirillov dimension.

Project supported by the Natural Science Foundation of China (No. 10801069).

(C)2008 American Mathematical Society 
Hopf algebras; that is, a finite dimensional Hopf algebra has global dimension 0 if and only if $\varepsilon\left(\int^{r}\right) \neq 0$, to some infinite dimensional case. They also use homological integrals to study infinite dimensional Hopf algebras of low Gelfand-Kirillov dimensions (denoted by GK-dimension for short), especially of GK-dimension 1, and ask the following question (Question 8.4 of $\underline{6}$ ):

$(Q *)$ Besides the group algebras $k \mathbb{Z}, k \mathbb{D}$ and infinite dimensional Taft algebras, are there some other examples of noetherian affine prime regular Hopf algebra of GK-dimension 1?

Here $\mathbb{D}$ is the infinite dihedral group. Note that in the commutative case, a prime ring is the same as an integral domain. Thus if $H$ is a commutative affine prime regular Hopf algebra of GK-dimension 1, then it is an affine integral regular commutative Hopf algebra of Krull dimension 1 and the corresponding affine variety is a connected algebraic group of dimension 1. Therefore, question $(Q *)$ generalizes the well-known facts about the classification of connected algebraic groups of dimension 1 (see Theorem 20.5 in [3]).

One of the main aims of this paper is to give an answer to $(Q *)$. A new example of a noetherian affine prime regular Hopf algebra of GK-dimension 1 is given in Section 2. For a noetherian affine prime regular Hopf algebra $H$ of GK-dimension 1 , it should fit into a short exact sequence (see (E0.2.2) of [6]):

$$
0 \rightarrow H_{c l} \rightarrow H \rightarrow H_{i q} \rightarrow 0,
$$

where $H_{c l}=H^{c o H_{i q}}$. The authors of [6] also ask the following question (Remark 7.2 (b) in [ $[$ ]):

$(Q * *) \quad$ Can $H_{c l}$ be equipped with a Hopf structure making the above exact sequence into a "twisted" short exact sequence of Hopf algebras?

The new example constructed in Section 2 will give a negative answer to this question.

Although this example makes the situation of noetherian affine prime regular Hopf algebras of GK-dimension 1 more complicated, the graded versions of them are the same. Indeed, denote the characteristic of $k$ by char $k$ and the integral order (for definition, see the paragraph before Lemma 3.2) of $H$ by $i o(H)$. Then we have the following result.

Theorem 1.1. Let $H$ be a noetherian affine prime regular Hopf algebra of GKdimension 1 such that $\operatorname{char} k \nmid i o(H)$. Then $\mathrm{gr} H$ is an infinite dimensional prime Taft algebra.

Here $\operatorname{gr} H$ is defined as follows. Let $H$ be a noetherian affine PI Hopf algebra and $\int^{r}$ be its right homological integral. For an $H$-module $M$, we denote $l . a n n_{H}(M)$ the set of left annihilators of $M$. It is an ideal. For two $H$-modules $M, N, M \otimes_{k} N$ is an $H$-module through the coproduct $\Delta$. We define $J_{i q}:=\bigcap_{p} l$.ann $n_{H}\left(\left(\int^{r}\right)^{\otimes p}\right)$, which is a Hopf ideal (see Sections 4, 6 of [6]). From this, we can construct its graded Hopf algebra $\operatorname{gr} H:=\bigoplus_{s \geq 0} J_{i q}^{s} / J_{i q}^{s+1}$.

This theorem improves Conjecture 2.1 in this paper and also gives a characterization to infinite dimensional prime Taft algebras; that is, infinite dimensional prime Taft algebras are graded noetherian affine prime regular Hopf algebras of GK-dimension 1. We refer to [9] for basic definitions and properties about general Hopf algebras and to 2, 6, 12, for some known results and for questions concerning the homological properties and homological integrals of noetherian affine PI Hopf algebras. 


\section{EXAMPLE}

For later use, we first introduce infinite dimensional Taft algebras.

Example 2.1 (Example 2.7 and Example 7.3 of [6]). Let $n, m$ and $t$ be integers and $q$ be an $n$-th primitive root of unity. Let $H$ be the $k$-algebra generated by $x$ and $g$ with relations

$$
g^{n}=1 \text { and } x g=q^{m} g x .
$$

The comultiplication $\Delta$, counit $\varepsilon$, and antipode $S$ are given by

$$
\begin{aligned}
\Delta(g) & =g \otimes g, \quad \Delta(x)=g^{t} \otimes x+x \otimes 1, \\
\varepsilon(g) & =1, \quad \varepsilon(x)=0, \\
S(g) & =g^{-1}, \quad S(x)=-g^{-t} x .
\end{aligned}
$$

This $H$ is called an infinite dimensional Taft algebra. It is easy to see that $H$ is prime if and only if $q^{m}$ is also an $n$-th primitive root of unity. It is a noetherian affine PI Hopf algebra of global dimension 1. The right homological integral of $H$ is isomorphic to $H /\left(x, g-q^{m}\right)$ as left $H$-modules. Thus, if $H$ is prime, then $i o(H)=n$ and $H_{i q} \cong H /(x)=k\langle g\rangle \cong k \mathbb{Z}_{n}$.

Next, we will construct our example. Consider the associative algebra $A(n, q)$ which is generated by $x, g$ and $g^{-1}$ with the relations

$$
g g^{-1}=g^{-1} g=1, \quad x g=q g x, x^{n}=1-g^{n},
$$

where $q \in k$ is an $n$-th primitive root of unity. The comultiplication, counit and antipode on $A(n, q)$ are defined by

$$
\begin{aligned}
\Delta(g) & =g \otimes g, \quad \Delta(x)=x \otimes 1+g \otimes x, \\
\varepsilon(g) & =1, \quad \varepsilon(x)=0, \\
S(g) & =g^{-1}, \quad S(x)=-g^{-1} x .
\end{aligned}
$$

For simplicity, we denote $A(n, q)$ by $A$ in this section.

Proposition 2.1. With operations defined as above, $A$ is a Hopf algebra.

Proof. The proof is standard. We decompose it into several steps.

Step 1 ( $\Delta$ is an algebra map). To show this, it is enough to show that

$$
\begin{aligned}
& \Delta(g) \Delta\left(g^{-1}\right)=\Delta\left(g^{-1}\right) \Delta(g)=1 \otimes 1, \\
& \Delta(x) \Delta(g)=q \Delta(g) \Delta(x), \\
& \Delta(x)^{n}=1 \otimes 1-g^{n} \otimes g^{n} .
\end{aligned}
$$

We only show (3) since the others are easy. For (3),

$$
\begin{aligned}
\Delta(x)^{n} & =(x \otimes 1+g \otimes x)^{n} \\
& =\sum_{i=0}^{n}\left(\begin{array}{c}
n \\
i
\end{array}\right)_{q} g^{n-i} x^{i} \otimes x^{n-i} \\
& =x^{n} \otimes 1+g^{n} \otimes x^{n}+\sum_{i=1}^{n-1}\left(\begin{array}{c}
n \\
i
\end{array}\right)_{q} g^{n-i} x^{i} \otimes x^{n-i} \\
& =x^{n} \otimes 1+g^{n} \otimes x^{n} \\
& =\left(1-g^{n}\right) \otimes 1+g^{n} \otimes\left(1-g^{n}\right)=1 \otimes 1-g^{n} \otimes g^{n} .
\end{aligned}
$$


Here $\left(\begin{array}{c}n \\ i\end{array}\right)_{q}$ is the Gaussian binomial coefficient which is defined by $\left(\begin{array}{c}n \\ i\end{array}\right)_{q}:=\frac{(n) !_{q}}{i !_{q}(n-i) !_{q}}$, where $i !_{q}=1_{q} \cdots i_{q}$ and $i_{q}=1+q+\cdots+q^{i-1}$. For the fourth equality we have used the fact that

$$
\left(\begin{array}{c}
n \\
i
\end{array}\right)_{q}=0 \quad \text { for } 1 \leq i \leq n-1 .
$$

Step 2 ( $\Delta$ is coassociative). By Step 1 , it is enough to check this for generators $g, g^{-1}, x$. That is, we need to show that

$$
(i d \otimes \Delta) \Delta(y)=(\Delta \otimes i d) \Delta(y)
$$

for $y=g, y=g^{-1}$ and $y=x$. But these are direct.

Step 3 ( $S$ is an algebra anti-homomorphism and $\varepsilon$ is an algebra morphism). To show that $S$ is an algebra anti-homomorphism, it is sufficient to verify the following identities:

$$
\begin{aligned}
& S(g) S\left(g^{-1}\right)=S\left(g^{-1}\right) S(g)=1, \\
& S(g) S(x)=q S(x) S(g), \\
& S(x)^{n}=1-g^{-n} .
\end{aligned}
$$

We only show (6) since the others are easy. In fact,

$$
\begin{aligned}
S(x)^{n} & =\overbrace{\left(-g^{-1} x\right) \cdot \ldots \cdot\left(-g^{-1} x\right)}^{n} \\
& =(-1)^{n} q^{-(1+\cdots+(n-1))} g^{-n} x^{n} \\
& =(-1)^{n} q^{-\frac{n(n-1)}{2}} g^{-n}\left(1-g^{n}\right) \\
& =(-1)^{n} q^{-\frac{n(n-1)}{2}}\left(g^{-n}-1\right) .
\end{aligned}
$$

Thus to show (6), we need to show that

$$
(-1)^{n} q^{-\frac{n(n-1)}{2}}=-1 .
$$

If $n$ is odd, (*) is clear. If $n$ is even,

$$
(-1)^{n} q^{-\frac{n(n-1)}{2}}=q^{-\left(n\left(\frac{n}{2}-1\right)+\frac{n}{2}\right)}=\frac{1}{q^{\frac{n}{2}}} .
$$

By $\left(q^{\frac{n}{2}}\right)^{2}=q^{n}=1$ and $q$ is an $n$-th primitive root of unity, $q^{\frac{n}{2}}=-1$. Therefore, $(*)$ is always true.

To show $\varepsilon$ is an algebra morphism, we need to show that

$$
\varepsilon(g) \varepsilon\left(g^{-1}\right)=\varepsilon\left(g^{-1}\right) \varepsilon(g)=1, \quad \varepsilon(x) \varepsilon(g)=q \varepsilon(g) \varepsilon(x), \text { and } \varepsilon(x)^{n}=0 .
$$

But these are easy.

Step 4. We have $(\varepsilon \otimes i d) \Delta(z)=z=(i d \otimes \varepsilon) \Delta(z), \quad z^{\prime} S\left(z^{\prime \prime}\right)=\varepsilon(z)=S\left(z^{\prime}\right) z^{\prime \prime}$ for $z \in A$ and $\Delta(z)=z^{\prime} \otimes z^{\prime \prime}$. Here we omit the summation notation for simplicity.

Indeed, by Step 3, we need only to check these equalities for $z=g, z=g^{-1}$ and $z=x$. But these are straightforward.

By Steps 1, 2, 3, 4, we know $A$ is a Hopf algebra.

For this Hopf algebra, the following lemma is obvious.

Lemma 2.2. A is noetherian affine. 
For any algebra $B$, we use $G K(B)$ to denote the GK-dimension of $B$.

Lemma 2.3. $A$ is $P I$ and $G K(A)=1$.

Proof. Clearly, $A$ is a finite module over $k\langle g\rangle \cong k \mathbb{Z}$ which is commutative of GKdimension 1. Thus $A$ is PI, and by Proposition 8.2.9 (ii) in [8],

$$
G K(A)=G K(k \mathbb{Z})=1 .
$$

For an algebra $B$ and a subset $I \subset B$, denote by $(I)$ the ideal generated by the set $I$. The global dimension of $B$ is denoted by gl.dim $B$ and for a $B$-module $N$,

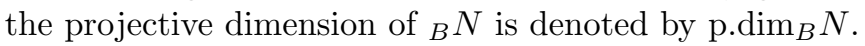

Lemma 2.4. gl. $\operatorname{dim} A=1$.

Proof. Denote by $M$ the trivial $A$-module $k$. By [5, it is enough to show that p.dim ${ }_{A} M=1$.

Clearly $x$ is a regular element (i.e. $x$ is not a zero divisor) of $A$. Since $x M=0$, $M$ cannot be a submodule of a free $A$-module and this implies $\operatorname{p} \cdot \operatorname{dim}_{A} M \neq 0$.

Let $I=(x)$. Since $x$ is a regular element of $A, A x \cong A$ as $A$-modules. This implies $\operatorname{p} \cdot \operatorname{dim}_{A}(A / I)=1$. Thus for any free $A / I$-module $F$, we have $\operatorname{p} \cdot \operatorname{dim}_{A} F=1$. Note that $A / I \cong k \mathbb{Z}_{n}$, which is semisimple (char $k \nmid n$ since we assume that we have an $n$-th primitive root of unity). Thus ${ }_{A / I} M$ is a direct summand of a free $A / I$-module, and hence $\operatorname{p.} \operatorname{dim}_{A} M=1$.

Recall that $J_{i q}=\bigcap_{p}$ l.ann ${ }_{H}\left(\left(\int^{r}\right)^{\otimes p}\right)$ for any Hopf algebra $H$ with homological integral. The following conclusion is Lemma 6.9 in [6].

Lemma 2.5. Let $H$ be a noetherian regular affine $P I$ Hopf algebra and $P_{0}$ the minimal prime ideal of $H$ contained in $\operatorname{Ker} \varepsilon$. If $G K(H)=1$, then $P_{0}=\bigcap_{m} J_{i q}^{m}$.

Lemma 2.6. $A$ is prime.

Proof. Here we freely use some of the notation in [6].

Note that $x$ is a normal element with $x h=\tau(h) x$ for all $h \in A$ and where $\tau: g \mapsto q g, x \mapsto x$. Since $A^{\prime}=A /(x)=k \mathbb{Z}_{n}, \int_{A^{\prime}}^{l}=k=A /(x, g-1)$. By Lemma 2.6 in [6],

$$
\int_{A}^{r}=S\left(\int_{A}^{l}\right)=S\left(\left(\int_{A^{\prime}}^{l}\right)^{\tau^{-1}}\right)=A /(x, g-q) .
$$

Thus l.ann $\int_{A}^{r}=(x, g-q)$. By direct computation, we can find that

$$
J_{i q}=\bigcap_{p} \operatorname{lann}_{A}\left(\left(\int^{r}\right)^{\otimes p}\right)=\left(x, g^{n}-1\right)=(x) .
$$

Clearly, $\bigcap_{m} J_{i q}^{m}=0$ and thus by Lemma $2.5, P_{0}=0$. Therefore, $A=A / P_{0}$ is prime since $P_{0}$ is a prime ideal.

Now we can give the main conclusion of this section.

Theorem 2.7. (i) $A$ is a noetherian affine prime regular Hopf algebra of GKdimension 1.

(ii) As a Hopf algebra, $A$ is not isomorphic to $k \mathbb{Z}, k \mathbb{D}$ or any one of the infinite dimensional Taft algebras. 
Proof. (i) is a direct consequence of Proposition 2.1, Lemma 2.2-2.4 and Lemma 2.6.

For (ii), consider the groups of all group-like elements of these Hopf algebras; we can find that only $k \mathbb{Z}$ has the same group as $A$. But, clearly, $k \mathbb{Z}$ is not isomorphic to $A$.

Remark 2.8. (i) Theorem 2.7 gives us a new example of a noetherian affine prime regular Hopf algebra of GK-dimension 1.

(ii) By the proof of Lemma 2.6, $A_{i q}:=A / J_{i q}=A /(x) \cong k \mathbb{Z}_{n}$. By direct computations, $A_{c l}:=A^{c o A_{i q}}$ is the subalgebra generated by $x, g^{n}$ and $g^{-n}$. By Theorem 7.1 in [6], $A_{c l}$ is a commutative domain of Krull dimension 1. We claim that we cannot equip it with a comultiplication which makes it into a Hopf algebra. Indeed, if we could, then the corresponding affine variety of $A_{c l}$ would be a connected algebraic group of dimension 1 . Thus

$$
A_{c l} \cong k[X] \text { or } A_{c l} \cong k\left[X^{ \pm 1}\right]
$$

since we have only two connected algebraic groups of dimension 1 (see Theorem 20.5 in [3). But these are clearly impossible. This means that we cannot give a Hopf structure on the algebra $A_{c l}$, and thus we give a negative answer to $(Q * *)$ given in the introduction.

(iii) We can generalize the example constructed above slightly. Let $t, n$ be natural numbers satisfying $(t, n)=1$. When the characteristic of $k$ is $p$, we also ask that $p \nmid t$. Define the Hopf algebra $A(t n, q)$ as follows.

As an associative algebra, $A(t n, q)$ is generated by $x, g$ and $g^{-1}$ subject to the relations

$$
g g^{-1}=g^{-1} g=1, \quad x g=q g x, x^{n}=1-g^{t n},
$$

where $q \in k$ is an $n$-th primitive root of unity. The comultiplication, counit and antipode on $A$ are defined by

$$
\begin{aligned}
\Delta(g) & =g \otimes g, \quad \Delta(x)=x \otimes 1+g^{t} \otimes x, \\
\varepsilon(g) & =1, \quad \varepsilon(x)=0, \\
S(g) & =g^{-1}, \quad S(x)=-g^{-t} x .
\end{aligned}
$$

Similarly, we can show that $A(t n, q)$ is also a noetherian affine prime regular Hopf algebra of GK-dimension 1.

The condition $p \nmid t$ is necessary when char $k=p$. Indeed, we can show that $A(n t, q)$ is not regular if $p \mid t$. To see this, we can assume that $A(n t, q)$ is regular. By Lemma 5.3 in [6], the global dimension of $A(n t, q)$ must be 1 . This implies that the submodule $A(n t, q) x+A(n t, q)(g-1)$ is projective. We denote this module by $N$. Thus we know that $N /(x) N$ is a projective $A(n t, q) /(x) \cong k \mathbb{Z}_{n t}$-module. It is easy to see that the image of $x$ in $N /(x) N$ is non-zero, and we denote this image by $x$ too. By $(q g-1) x=x(g-1) \in(x) N, x$ generates a 1-dimensional $k \mathbb{Z}_{n t}$-submodule of $N /(x) N$. By a direct computation, we can also see that this 1-dimensional submodule is a direct summand of $N /(x) N$. This means that we have a projective $k \mathbb{Z}_{n t}$-module of dimension 1 . This is absurd.

Conjecture 2.1. For a noetherian affine prime regular Hopf algebra $H$ of GKdimension 1, $H$ is isomorphic to one of the following Hopf algebras:

$k \mathbb{Z}, k \mathbb{D}$, infinite dimenisonal Taft algebras, $A(t n, q)$. 


\section{Proof of Theorem 1.1}

Of course, Theorem 1.1 improves Conjecture 2.1. To prove this theorem, we need to recall the definition and some properties of the biproduct (or bosonization, in Majid's terminology).

Let $H, H_{0}$ be Hopf algebras and $\pi: H \rightarrow H_{0}$ and $\iota: H_{0} \rightarrow H$ Hopf homomorphisms. Assume that $\pi \iota=i d_{H_{0}}$, so that $\pi$ is surjective and $\iota$ is injective. Define

$$
R_{H}:=H^{c o \pi}=\{h \in H \mid(i d \otimes \pi) \Delta(h)=h \otimes 1\} .
$$

By a result of Radford (see Theorem 3 of [10]),

$$
H \cong R_{H} \times H_{0} \text { as Hopf algebras, }
$$

where " $\times$ " was called the biproduct in [10] and bosonization in [7]. Knowledge about the biproduct or bosonization can be found in 1, 17, 10. We list some facts, for which we will not give any proof, from [1, 7], 10]:

(Fact i): $R_{H}$ is a braided Hopf algebra in ${ }_{H_{0}}^{H_{0}} \mathcal{Y D}$, the category of Yetter-Drinfeld modules over $H_{0}$ (see [1]). The action · of $H_{0}$ on $R_{H}$ is the restriction of the adjoint action and the coaction is $(\pi \otimes i d) \Delta$;

(Fact ii): $R_{H}=\left\{h^{\prime} \iota \pi S\left(h^{\prime \prime}\right) \mid h \in H\right\}$ and the comultiplication in $R_{H}$ is given by $\Delta_{R_{H}}(r)=r^{\prime} \iota \pi S\left(r^{\prime \prime}\right) \otimes r^{\prime \prime \prime}$ for $r \in R_{H}$;

(Fact iii): As a linear space, $R_{H} \times H_{0}=R_{H} \otimes H_{0}$. Its multiplication and comultiplication are the usual smash product and smash coproduct respectively. That is, if we denote the comultiplication of $R_{H}$ by $\Delta_{R_{H}}$ and write $\Delta_{R_{H}}(r)=$ $r^{(1)} \otimes r^{(2)}$, then for $r, s \in R_{H}$ and $h, f \in H_{0}$,

$$
\begin{aligned}
& (r \times h)(s \times f)=r\left(h^{\prime} \cdot s\right) \times h^{\prime \prime} f, \\
& \Delta(r \times h)=r^{(1)} \times\left(r^{(2)}\right)_{(-1)} h^{\prime} \otimes\left(r^{(2)}\right)_{(0)} \times h^{\prime \prime} .
\end{aligned}
$$

Now let $H$ be a noetherian affine PI Hopf algebra and $\operatorname{gr} H=\bigoplus_{s \geq 0} J_{i q}^{s} / J_{i q}^{s+1}$. The following lemma implies that gr $H$ is a Hopf algebra too.

Lemma 3.1. Let $H$ be a Hopf algebra and $I \triangleleft H$ be an ideal of $H$. Then gr $H:=$ $H / I \oplus I / I^{2} \oplus \cdots$ is a Hopf algebra if and only if $I$ is a Hopf ideal.

Proof. The same conclusion has been proved in [4] when $H$ is finite dimensional and $I$ is taken to be the Jacobson radical of $H$ (see Lemma 5.1 in [4]). It is straightforward to see that the proof given in [4] can be applied to our case directly.

Let $\iota: H / J_{i q} \hookrightarrow \bigoplus_{s \geq 0} J_{i q}^{s} / J_{i q}^{s+1}$ be the inclusion and let $\pi: \bigoplus_{s \geq 0} J_{i q}^{s} / J_{i q}^{s+1} \rightarrow$ $H / J_{i q}$ be the projection with kernel $\bigoplus_{s>0} J_{i q}^{s} / J_{i q}^{s+1}$. Clearly, $\pi$ is a Hopf algebra retraction of $\iota$. Denote $H_{i q}:=H / J_{i q}$ and $R_{\operatorname{gr} H}:=H^{c o H_{i q}}=\{h \in \operatorname{gr} H \mid(i d \otimes$ $\pi) \Delta(h)=h \otimes 1\}$. Then by the discussions above,

$$
\operatorname{gr} H \cong R_{\mathrm{gr} H} \times H_{i q} .
$$

Let $\int^{r}$ be the right homological integral of $H$. The integral order of $H$, denoted by $i o(H)$, is defined to be the minimal positive integer $n$ (or $\infty$ if no such $n$ ) such that $\left(\int^{r}\right)^{\otimes n} \cong k$ as left $H$-modules, where $k$ denotes the trivial $H$-module. The following lemma is the combination of Lemma 4.4 and Lemma 5.3 in [6]. 
Lemma 3.2. Let $H$ be a noetherian regular affine PI Hopf algebra. Then

(a) io $(H)$ is finite, say $n$, and

(b) if char $k \nmid i o(H)$, then $H_{i q} \cong k G$ where $G$ is the cyclic group of order $n$.

Proof of Theorem 1.1. Let $H$ be a noetherian affine prime regular Hopf algebra of GK-dimension 1 (it is PI automatically [11]). Then $\operatorname{gr} H \cong R_{\operatorname{gr} H} \times H_{i q}$. By Lemma 3.2, its integral order is finite, $n$ say, and $H_{i q}=k G$ where $G=\left\langle g \mid g^{n}=1\right\rangle$. By Lemma 7.6 (b) and the proof of Proposition 7.7 (e) in [6], we know that $R_{\operatorname{gr} H}=$ $(\operatorname{gr} H)^{c o H_{i q}}=(\operatorname{gr} H)^{k G} \cong k[x]$.

By (Fact i), $k[x]$ is a braided Hopf algebra in ${ }_{k G}^{k G} \mathcal{Y D}$. Thus $k[x]$ is a coalgebra. Note that (Fact ii) implies that $k[x]$ is indeed a graded, braided Hopf algebra in ${ }_{k G}^{k G} \mathcal{Y D}$ since gr $H$ is graded. In particular, the comultiplication of $k[x]$ is graded, which implies $\Delta_{k[x]}(x)=x \otimes 1+1 \otimes x$. By the definition of coaction (see (Fact i)), we know $k x$ is a $k G$-comodule. Therefore, $\rho(x):=x_{(-1)} \otimes x_{(0)}=g^{t} \otimes x$ for some $t$. By (Fact iii), $\Delta_{\text {gr } H}(x \times 1)=x^{(1)} \times\left(x^{(2)}\right)_{(-1)} \otimes\left(x^{(2)}\right)_{(0)} \times 1=(x \times 1) \otimes(1 \times 1)+$ $\left(1 \times g^{t}\right) \otimes(x \times 1)$. This means that $\Delta_{\operatorname{gr} H}(x)=x \otimes 1+g^{t} \otimes x$ if we write $s \times h$ by sh for simplicity.

By (Fact i), we know $k x$ is a $k G$-module and $g \cdot x=g x g^{-1}$. This implies $g x g^{-1}=q x$ for some $n$-th root of unity $q \in k$. Since $\operatorname{gr} H$ is prime (Proposition 7.7 (d) in [6]), $q$ is a primitive $n$-th root of unity.

Take an infinite dimensional Taft algebra $T_{q, t}$ which is generated by $y$ and $h$ with relations $h^{n}=1$ and $h y=q y h$. Its comultiplication is defined to be $\Delta(h)=h \otimes h$ and $\Delta(y)=y \otimes 1+h^{t} \otimes y$. Define the map $f: T_{q, t} \rightarrow \operatorname{gr} H$ by $h \mapsto g$ and $y \mapsto x$. Then clearly $f$ is a surjective Hopf morphism. Since $f$ is injective in the first term of the coalgebra filtration, $f$ is injective (see Lemma 5.3.3 in 9]). Thus $\operatorname{gr} H$ is isomorphic to an infinite dimensional Taft algebra.

Remark 3.3. By Theorem 1.1, the class of infinite dimensional prime Taft algebras is just the class of graded (with respect to the integral quotient ideal $J_{i q}$ ) noetherian affine prime regular Hopf algebras of GK-dimension 1.

Example 3.1. Let $\mathbb{D}=\left\langle g, x \mid g^{2}=1, g x g^{-1}=x^{-1}\right\rangle$ be the infinite dihedral group. In this example, we show that grkD is indeed an infinite dimensional Taft algebra. Similarly, we can check Theorem 1.1 for $A(n, q)$. By Example 4.6 in [6], the group algebra $k \mathbb{D}$ is a noetherian affine prime regular Hopf algebra of GK-dimension 1 and $J_{i q}=(x-1)$. Thus gr $k \mathbb{D}=k \mathbb{D} /(x-1) \oplus(x-1) /(x-1)^{2} \oplus \cdots$. Clearly, in $\operatorname{gr} k \mathbb{D}, x-1$ is a non-zero vector which belongs to the part $J_{i q} / J_{i q}^{2}$. Similarly, since $J_{i q}=\left(x^{-1}-1\right), x^{-1}-1$ is a non-zero vector which also belongs to the part $J_{i q} / J_{i q}^{2}$. By $x-1+x^{-1}-1=-2+x+x^{-1}=\left(1-x^{-1}\right)(x-1)$, which belongs to $J_{i q}^{2}, x-1+x^{-1}-1=0$ in $J_{i q} / J_{i q}^{2}$. Thus, in $\operatorname{gr} k \mathbb{D},(x-1)+\left(x^{-1}-1\right)=0$, which implies $x^{-1}=-x+2$. By the relation $g x=x^{-1} g$ in $k \mathbb{D}$, we have $g x=-x g+2 g$ in $\operatorname{gr} k \mathbb{D}$.

Denote $x-1$ by $y$. Then

$$
g y=g(x-1)=g x-g=-x g+2 g-g=-x g+g=-(x-1) g=-y g
$$

and $\Delta(y)=\Delta(x-1)=(x-1) \otimes x+1 \otimes(x-1)$. But $x \equiv 1(\bmod (x-1))$. Thus, in $\operatorname{gr} k \mathbb{D}$,

$$
\Delta(y)=y \otimes 1+1 \otimes y .
$$

By the discussions above, gr $k \mathbb{D} \cong k[y] \times k \mathbb{Z}_{2}$ is an infinite dimensional Taft algebra. 


\section{ACKNOWLEDGEMENTS}

This work was finished when the author visited Oxford University with financial support from the Leverhulme Trust through the Academic Interchange Network Algebras, Representations and Applications. I would like to thank the university for their hospitality and the Leverhulme Trust for its financial support. I would also like to thank the referee for very valuable comments. Especially, I want to thank Professor Ken A. Brown for his example, which helped me to correct an error in this paper.

\section{REFERENCES}

1. N. Andruskiewitsch and H.-J. Schneider, Pointed Hopf Algebras, in "New directions in Hopf algebras", 1-68, Math. Sci. Res. Inst. Publ. 43, Cambridge Univ. Press, Cambridge, 2002. MR.1913436 (2003e:16043)

2. K. A. Brown and K. R. Goodearl, Homological Aspects of Noetherian PI Hopf Algebras of Irreducible Modules and Maximal Dimension, J. Algebra 198 (1997), 240-265. MR 1482982 (99c:16036)

3. J. E. Humphreys, Linear Algebraic Groups, Graduate Texts in Math. 21, Springer-Verlag, New York-Heidelberg-Berlin, 1975. MR0396773 (53:633)

4. G. Liu, On the Structure of Tame Graded Basic Hopf Algebras, J. Algebra 299 (2006), 841-853. MR 2228342 (2007b:16083)

5. M. E. Lorenz and M. Lorenz, On Crossed Products of Hopf Algebras, Proc. Amer. Math. Soc. 123(1) (1995), 33-38. MR:1227522 (95c:16014)

6. D.-M. Lu, Q.-S. Wu and J. J. Zhang, Homological Integral of Hopf Algebras, Trans. Amer. Math. Soc. 359 (2007), 4945-4975. MR2320655 (2008f:16083)

7. S. Majid, Cross Products by Braided Groups and Bosonization, J. Algebra 163 (1994), 165190. MR1257312 (94m:18009)

8. J. C. McConnell and J. C. Robson, Noncommutative Noetherian Rings, Wiley, Chichester, 1987. MR $934572(89 \mathrm{j}: 16023)$

9. S. Montgomery, Hopf Algebras and Their Actions on Rings. CBMS Regional Conference Series in Math., vol. 82, Amer. Math. Soc., Providence, RI, 1993. MR1243637 (94i:16019)

10. D. Radford, The Structure of Hopf Algebras with a Projection, J. Algebra 92 (1985), 322-347. MR 778452 (86k:16004)

11. L. W. Small, J. T. Stafford and R. B. Warfield Jr., Affine Algebras of Gelfand-Kirillov Dimension One Are PI, Math. Proc. Cambridge Philos. Soc. 97 (1985), no. 3, 407-414. MR7786674 (86g:16025)

12. Q.-S. Wu and J. J. Zhang, Noetherian PI Hopf Algebras Are Gorenstein, Trans. Amer. Math. Soc. 355 (2003), no. 3, 1043-1066. MR1938745 (2003m:16056)

Institute of Mathematics, Academy of Mathematics and Systems Science, Chinese Academy of Sciences, Beijing 100080, People's Republic of China

Current address: Department of Mathematics, Nanjing University, Nanjing 210093, People's Republic of China

E-mail address: gxliu@nju.edu.cn 